\title{
Model for Solving Fuzzy Multiple Objective Problem
}

\author{
Ritika Chopra ${ }^{1}$, Ratnesh R. Saxena ${ }^{2}$ \\ ${ }^{1}$ Department of Mathematics, Delhi University, Delhi, India \\ ${ }^{2}$ Department of Mathematics, Deen Dayal Upadhyay College, Delhi University, Delhi, India \\ Email: ritikaritsin17@gmail.com
}

Received November 27, 2012; revised December 25, 2012; accepted January 10, 2013

\begin{abstract}
In real world decision making problems, the decision maker has to often optimize more than one objective, which might be conflicting in nature. Also, it is not always possible to find the exact values of the input data and related parameters due to incomplete or unavailable information. This work aims at developing a model that solves a multi objective distribution programming problem involving imprecise available supply, forecast demand, budget and unit cost/profit coefficients with triangular possibility distributions. This algorithm aims to simultaneously minimize cost and maximize profit with reference to available supply constraint at each source, forecast demand constraint at each destination and budget constraint. An example is given to demonstrate the functioning of this algorithm.
\end{abstract}

Keywords: Decision Making Problems; Multi Objective Distribution Programming Problem; Fuzzy Set Theory

\section{Introduction}

The distribution planning decision (DPD) problem involves optimizing the distribution plan for transporting goods from a set of sources to a set of destinations in a supply chain. With a variety of distributing routes, the aim of the DPD problem is to determine how many units should be shipped from one source to one destination so that the available supply at each source and the forecast demand at each destination are satisfied. In most realworld DPD problems, the decision maker (DM) must simultaneously handle conflicting objectives like minimizing total distribution costs, number of rejected items and delivery time/distance, and maximizing total profits, relative safety and customer service level.

When any of the ordinary linear programming (LP) method or the existing solution algorithms is used to solve DPD problems, the objectives and model inputs are generally assumed to be precisely given (crisp). However, in practical DPD problems, input data and related parameters, such as available supply, forecast demand and related cost/time/profit coefficients, are often imprecise (fuzzy) because of incomplete or (and) unavailable information. So, conventional LP method and solution algorithms cannot solve the imprecise (fuzzy) DPD programming problems.

Fuzzy set theory was presented by Zadeh [1] and has been applied extensively in various fields. His study showed that the importance of the theory of possibility is based on the fact that much of the information, on which human decisions are based, is possibilistic rather than probabilistic in nature. In 1976, Zimmermann [2] first introduced fuzzy set theory into an ordinary LP problem with fuzzy objective and constraints. Following, the fuzzy decision making method proposed by Bellman and Zadeh [3] confirmed the existence of an equivalent ordinary LP form. Subsequently, Chanas et al. [4] presented a fuzzy linear programming (FLP) method to solve DPD problems with crisp cost coefficients and fuzzy supply and demand. Furthermore, Zimmermann [5] extended his FLP method to a multi-objective linear programming (MOLP) problem. In addition, researchers have developed several FGP methods to solve multi-objective DPD problems. Li and Lai [6] designed a fuzzy compromise programming method to obtain a non-dominated compromise solution for multi-objective DPD problems in which various objectives were synthetically considered with the marginal evaluation for individual objectives and the global evaluation for all objectives. Related studies on solving DPD problems with multiple fuzzy objectives included Hussein [7] and Abd El-Wahed [8]. Moreover, Lai and Hwang [9] developed an auxiliary MOLP model for solving PLP problems with imprecise objective and/or constraint coefficients with triangular distributions. Tien-Fu Liang [10] integrated the available concepts to solve multi-objective DPD problems involveing imprecise available supply, forecast demand and unit cost/time coefficients with triangular possibility distributions.

In this article, we aim to solve a multi objective distribution programming problem which simultaneously minimizes cost and maximizes profit with reference to available supply constraint at each source, forecast de- 
mand constraints at each destination and the total budget constraint. We have assumed the available supply, forecast demand, budget and related cost and profit coefficients to be imprecise with triangular possibility distributions. Also, the interactive PLP method provides a systematic framework that facilitates the decision making process, enabling a DM to interactively modify the imprecise data and related parameters until a satisfactory solution is obtained. The proposed methodology can be applied to various real world multi objective problems such as assignment problems, transportation problems and many more such problems in which the information is given in the form of triangular possibility distributions.

\section{Problem Description}

A general multi objective distribution programming problem aims to determine the right plan for distributing a homogeneous commodity from $m$ sources to $n$ destinations. Each source has an available supply of the commodity to distribute to various destinations, and each destination has a forecast demand of the commodity to be received from various sources. The available supply for each source, the forecast demand for each destination, and related cost/profit coefficients are generally imprecise over the planning horizon due to incomplete and/or unobtainable information. The proposed PLP method aims to simultaneously minimize the total distribution costs and maximize the total profit with reference to available supply constraint at each source, forecast demand constraint at each destination and the total budget constraint. This work focuses on developing an interactive PLP method for optimizing the distribution plan in uncertain environments.

Thus, all the objectives are imprecise thereby incorporating the variations in the decision maker's judgments relating to the solutions of the multi objective optimization problems in a framework of fuzzy aspiration levels. The pattern of triangular possibility distribution is adopted to represent the imprecise objective functions and related imprecise numbers. All of the objective functions and the constraints are linear and the cost and profit on a given route are directly proportional to the units distributed.

\section{Notations}

\subsection{Index Sets}

- $i$ index for source, for all $i=1,2, \cdots, m$;

- $j$ index for destination, for all $j=1,2, \cdots, n$;

- $g$ index for objectives, for $g=1,2$.

\subsection{Decision Variables}

- $x_{i j}$ units distributed from source $i$ to destination $j$ (units).

\subsection{Objective Functions}

- $\widetilde{Z}_{1}$ total distribution costs (\$);

- $\widetilde{Z}_{2}$ total profit (\$).

\subsection{Parameters}

- $\widetilde{c_{i j}}$ distribution cost per unit delivered from source i to destination $j$ (\$/unit);

- $\quad \widetilde{p_{i j}}$ profit per unit delivered from source $i$ to destination $j$ (\$/unit);

- $\quad \widetilde{S}_{i}$ total available supply for each source $i$ (units);

- $\widetilde{D_{j}}$ total forecast demand of each destination $j$ (units);

- $\tilde{B}$ total budget $(\$)$.

\section{Problem at a Glance}

The aim of the problem is to minimize total cost of distribution and maximize profit (where cost and profit coefficients are fuzzy numbers with triangular possibility distributions) with reference to imprecise available supply constraint at each source, forecast demand constraint at each destination and the total budget constraint. Mathematically,

$$
\begin{aligned}
& \min \widetilde{z_{1}}=\sum_{i=1}^{m} \sum_{j=1}^{n} \widetilde{c_{i j}} x_{i j} \\
& \max \widetilde{z_{2}}=\sum_{i=1}^{m} \sum_{j=1}^{n} \widetilde{p_{i j}} x_{i j}
\end{aligned}
$$

Subject to

$$
\begin{aligned}
& \sum_{j=1}^{n} x_{i j} \leq \widetilde{S_{i}}, \forall i=1,2, \cdots, m \\
& \sum_{i=1}^{m} x_{i j} \leq \widetilde{D_{j}}, \forall j=1,2, \cdots, n \\
& \sum_{j=1}^{n} \sum_{i=1}^{m} \widetilde{c_{i j}} x_{i j} \leq \tilde{B} x_{i j} \geq 0, \\
& \forall i=1,2, \cdots, m, \forall j=1,2, \cdots, n
\end{aligned}
$$

\section{Methodology}

This work assumes the DM to have already adopted the pattern of triangular possibility distribution for all imprecise numbers. For instance, the triangular possibility distribution of imprecise cost coefficient (given in Figure 1), $\widetilde{c_{i j}}=\left(c_{i j}^{o}, c_{i j}^{m}, c_{i j}^{p}\right)$, where $c_{i j}^{m}$ is the most possible value that definitely belongs to the set of available values (degree of association $=1$, if normalized), $c_{i j}^{o}$ is the most optimistic value that has a very low likelihood of belonging to the set of available values (degree of association $=0$ ) and $c_{i j}^{p}$ is the most pessimistic value that has a very low likelihood of belonging to the set of available values (degree of association $=0$ ). 


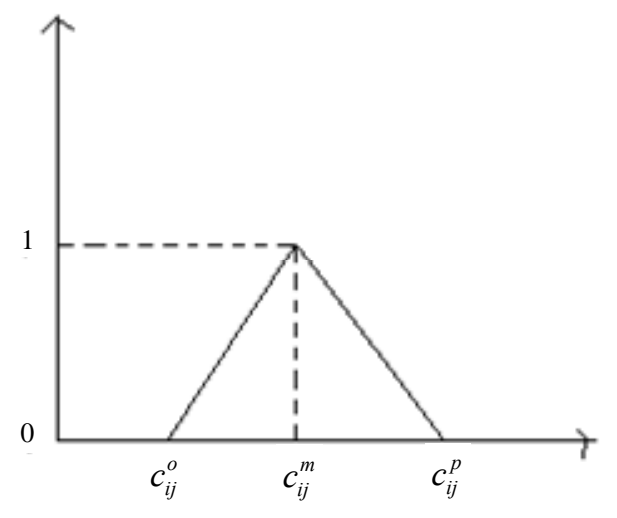

Figure 1. Triangular possibility distribution of $\widetilde{c_{i j}}$.

Similarly, triangular possibility distribution of imprecise profit coefficient, $\widetilde{p_{i j}}=\left(p_{i j}^{p}, p_{i j}^{m}, p_{i j}^{o}\right)$, where $p_{i j}^{m}$ is the most possible value that definitely belongs to the set of available values ( degree of association $=1$, if normalized), $p_{i j}^{o}$ is the most optimistic value that has a very low likelihood of belonging to the set of available values ( degree of association $=0$ ) and $p_{i j}^{p}$ is the most pessimistic value that has a very low likelihood of belonging to the set of available values ( degree of association $=0$ ).

Geometrically, these imprecise objective functions are fully defined by three prominent points $\left(z^{m}, 1\right),\left(z^{o}, 0\right)$ and $\left(z^{p}, 0\right)$. The imprecise cost objective can be minimized by pushing the three prominent points of triangular possibility distribution towards left and the imprecise profit objective can be maximized by pushing these points to the right. Because of the vertical coordinates of the prominent points being fixed at either 1 or 0 , the three horizontal coordinates are the only considerations. Thus we aim to simultaneously minimize the most possible objective value of the imprecise cost objective function, $z_{1}^{m}$, maximize the possibility of obtaining lower objective value, $\left(z_{1}^{m}-z_{1}^{o}\right)$ (region I of the possibility distribution in Figure 2), and minimize the risk of obtaining higher objective value, $\left(z_{1}^{p}-z_{1}^{m}\right)$ (region II of the possibility distribution in Figure 2) and maximize the most possible objective value of the imprecise profit objective function, $z_{2}^{m}$, minimize the possibility of obtaining lower objective value, $\left(z_{2}^{m}-z_{2}^{p}\right)$ (i.e. region I) and maximize the possibility of obtaining higher objective value, $\left(z_{2}^{o}-z_{2}^{m}\right)$ (i.e. region II).

Therefore, the above two fuzzy objectives are broken down into the following six crisp objectives

$$
\begin{aligned}
& \min z_{11}=\sum_{j=1}^{n} \sum_{i=1}^{m} c_{i j}^{m} x_{i j} \\
& \max z_{12}=\sum_{i=1}^{m} \sum_{j=1}^{n}\left(c_{i j}^{m}-c_{i j}^{o}\right) x_{i j}
\end{aligned}
$$

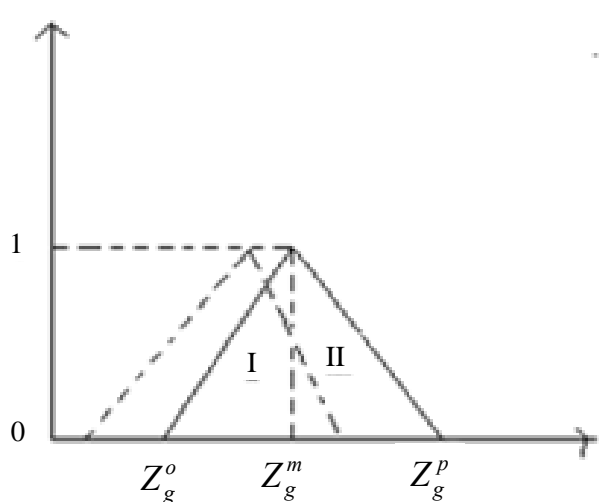

Figure 2. Strategy involved.

$$
\begin{aligned}
& \min z_{13}=\sum_{i=1}^{m} \sum_{j=1}^{n}\left(c_{i j}^{p}-c_{i j}^{m}\right) x_{i j} \\
& \max z_{21}=\sum_{i=1}^{m} \sum_{j=1}^{n} p_{i j}^{m} x_{i j} \\
& \min z_{22}=\sum_{i=1}^{m} \sum_{j=1}^{n}\left(p_{i j}^{m}-p_{i j}^{p}\right) x_{i j} \\
& \max z_{23}=\sum_{i=1}^{m} \sum_{j=1}^{n}\left(p_{i j}^{o}-p_{i j}^{m}\right) x_{i j}
\end{aligned}
$$

Further, the imprecise available supply $\widetilde{S}_{i}$, at each source and the imprecise forecast demand, $\widetilde{D_{j}}$, at each destination have triangular possibility distributions with the most and least possible values. $\widetilde{S}_{i}$ and $\widetilde{D_{j}}$ are converted to crisp numbers by applying the weighted sum approach. Let the minimum acceptable possibility, $\beta$, be given by the DM. Then the corresponding auxiliary inequality constraints can be represented as follows

$$
\begin{aligned}
& \sum_{j=1}^{n} x_{i j} \leq w_{1} S_{i, \beta}^{o}+w_{2} S_{i, \beta}^{m}+w_{3} S_{i, \beta}^{p}, \forall i=1,2, \cdots, m \\
& \sum_{i=1}^{m} x_{i j} \geq w_{1} D_{i, \beta}^{o}+w_{2} D_{i, \beta}^{m}+w_{3} D_{i, \beta}^{p}, \forall j=1,2, \cdots, n
\end{aligned}
$$

where, $w_{1}+w_{2}+w_{3}=1, \quad w_{1}, w_{2}$ and $w_{3}$ represent the weights of the most possible values, most optimistic values and the most pessimistic values of the imprecise supply demand, respectively. Related methods and algorithms for determining weights include the analytic hierarchy process (AHP) and direct assessment methods. In real-world DPD problems, detailed study of the effects of various weighting methods should be based on a DM's experience and knowledge.

Additionally, in the total budget constraint, the given imprecise budget has triangular possibility distribution with the most possible and least possible values. The imprecise inequality corresponding to the total budget is defuzzified using fuzzy ranking concept. Accordingly, if 
the minimum acceptable possibility is specified by the $\mathrm{DM}$, the corresponding auxiliary crisp inequality can be represented as

$$
\begin{gathered}
\sum_{i=1}^{m} \sum_{j=1}^{n} c_{i j}^{m} x_{i j} \leq B_{\beta}^{m} \\
\sum_{i=1}^{m} \sum_{j=1}^{n} c_{i j}^{o} x_{i j} \leq B_{\beta}^{o} \\
\sum_{i=1}^{m} \sum_{j=1}^{n} c_{i j}^{p} x_{i j} \leq B_{\beta}^{p}
\end{gathered}
$$

The auxiliary MOLP problem developed above can be converted into an equivalent ordinary LP form using Zimmermann's linear membership functions [2] to represent all of the fuzzy objectives of the DM, together with the fuzzy decision-making concept of Bellman and Zadeh [3]. First, obtain the corresponding positive ideal solutions (PIS) and negative ideal solutions (NIS) of all the crisp objective functions of the auxiliary MOLP problem, as follows

$$
\begin{aligned}
& z_{11}^{P I S}=\min z_{1}^{m}, z_{12}^{P I S}=\max \left(z_{1}^{m}-z_{1}^{o}\right), \\
& z_{13}^{P I S}=\min \left(z_{1}^{p}-z_{1}^{m}\right) ; \\
& z_{21}^{P I S}=\max z_{2}^{m}, z_{22}^{P I S}=\min \left(z_{2}^{m}-z_{2}^{p}\right), \\
& z_{23}^{P I S}=\max \left(z_{2}^{o}-z_{2}^{m}\right) ; \\
& z_{11}^{N I S}=\max z_{1}^{m}, z_{12}^{N I S}=\min \left(z_{1}^{m}-z_{1}^{o}\right), \\
& z_{13}^{N I S}=\max \left(z_{1}^{p}-z_{1}^{m}\right) ; \\
& z_{21}^{N I S}=\min z_{2}^{m}, z_{22}^{N I S}=\max \left(z_{2}^{m}-z_{2}^{p}\right), \\
& z_{23}^{N I S}=\min \left(z_{2}^{o}-z_{2}^{m}\right) .
\end{aligned}
$$

Furthermore, the corresponding linear membership function for each of the new objective functions of the auxiliary MOLP problem is defined by

$$
f_{11}\left(z_{11}\right)= \begin{cases}1 & \text { if } z_{11} \leq z_{11}^{P I S} \\ \frac{z_{11}^{N I S}-z_{11}}{z_{11}^{N I S}-z_{11}^{P I S}} & \text { if } z_{11}^{P I S} \leq z_{11} \leq z_{11}^{N I S} \\ 0 & \text { if } z_{11}^{N I S} \leq z_{11}\end{cases}
$$

$$
f_{12}\left(z_{12}\right)= \begin{cases}1 & \text { if } z_{12} \leq z_{12}^{P I S} \\ \frac{z_{12}^{N I S}-z_{12}}{z_{12}^{N I}-z_{12}^{P I S}} & \text { if } z_{12}^{P I S} \leq z_{12} \leq z_{12}^{N I S} \\ 0 & \text { if } z_{12}^{N I S} \leq z_{12}\end{cases}
$$

Linear membership functions for objective functions (5.3) and (5.5) are similar to the linear membership function for objective function (5.1) given by the Equation (5.12) and the linear membership function for objective functions (5.4) and (5.5) are similar to the linear membership function for objective function (5.2) given by the Equation (5.13).

Finally, aggregate all fuzzy sets using the minimum operator of fuzzy decision making concept. Let $\lambda$ be the auxiliary variable $(0 \leq \lambda \leq 1)$, which represents the degree of satisfaction of the decision maker. Then, the corresponding single objective linear programming problem is $\max \lambda$

$$
\begin{aligned}
& \text { s.t } f_{g 1}\left(z_{g 1}\right) \geq \lambda, g=1,2 ; f_{g 2}\left(z_{g 2}\right) \geq \lambda, g=1,2 ; \\
& f_{g 3}\left(z_{g 3}\right) \geq \lambda, g=1,2 \\
& \sum_{j=1}^{n} x_{i j} \leq w_{1} S_{i, \beta}^{o}+w_{2} S_{i, \beta}^{m}+w_{3} S_{i, \beta}^{p}, \forall i=1,2, \cdots, m \\
& \sum_{i=1}^{m} x_{i j} \geq w_{1} D_{i, \beta}^{o}+w_{2} D_{i, \beta}^{m}+w_{3} D_{i, \beta}^{p}, \forall j=1,2, \cdots, n \\
& \sum_{i=1}^{m} \sum_{j=1}^{n} c_{i j}^{m} x_{i j} \leq B_{\beta}^{m}, \sum_{i=1}^{m} \sum_{j=1}^{n} c_{i j}^{o} x_{i j} \leq B_{\beta}^{o}, \sum_{i=1}^{m} \sum_{j=1}^{n} c_{i j}^{p} x_{i j} \leq B_{\beta}^{p}, \\
& x_{i j} \geq 0, \forall i=1,2, \cdots, m, \forall j=1,2, \cdots, n .
\end{aligned}
$$

The above problem is a crisp single objective linear programming problem which can easily be solved using MATLAB.

\section{Numerical Illustration}

Consider the problem given in Table 1. Also, the total budget,

Table 1. Problem in consideration.

\begin{tabular}{cccccc}
\hline & A & B & C & D & Supply (in thousand) \\
\hline \multirow{2}{*}{1} & $(0.6,0.8,0.9)^{* *}$ & $(2.4,3.0,3.5)$ & $(1.8,2.1,2.3)$ & $(1.5,1.8,2.0)$ & $(17.2,18.0,19.2)$ \\
& $(0.1,0.3,0.4)^{* *}$ & $(1.0,1.5,2.0)$ & $(1.0,1.4,1.6)$ & $(0.7,0.9,1.0)$ & \\
2 & $(1.1,1.3,1.4)$ & $(3.1,3.6,4.1)$ & $(1.4,1.6,1.7)$ & $(2.2,2.5,2.7)$ & $(22.6,24.0,25.8)$ \\
& $(0.4,0.6,0.7)$ & $(1.5,1.8,2.1)$ & $(0.8,0.9,1.1)$ & $(1.0,1.4,1.8)$ & \\
& $(1.5,1.8,2.0)$ & $(3.1,3.5,3.9)$ & $(2.1,2.4,2.7)$ & $(0.8,1.0,1.1)$ & $(12.6,13.0,13.6)$ \\
& $(0.6,0.9,1.1)$ & $(1.4,1.8,2.2)$ & $(1.3,1.5,1.7)$ & $(0.2,0.3,0.4)$ & \\
\hline
\end{tabular}

*Denotes the cost coefficient; ${ }^{* *}$ Denotes the profit coefficient. 


$$
\tilde{B}=\$(132000,140000,148000) .
$$

First, formulate the original multi-objective fuzzy problem using Equations (4.1) to (4.5). Then, develop the new objective functions for the new crisp multi-objective problem using Equations (5.1) to (5.6). Further, defuzzify fuzzy constraints to get crisp constraints using Equations (5.7) to (5.11) at $\beta=0.5$ assuming $w_{1}=w_{3}=1 / 4$ and $w_{2}=1 / 2$.

Get the PIS and NIS values (given in Table 2 below) corresponding to all the crisp objectives.

Additionally, the corresponding linear membership function for each of the fuzzy objectives in the auxiliary MOLP can be defined using Equations (5.12) and (5.13). Finally, the auxiliary MOLP can be converted to an equivalent LP model using the minimum operator to aggregate all fuzzy sets. This LP is then solved using MATLAB.

The solutions are imprecise and have triangular possibility distribution, and overall degree of decision maker satisfaction is 0.9861 . Thus, the optimal plan by the above method is as follows:

$$
\begin{aligned}
& x_{11}=10211, x_{12}=00667, x_{13}=00002, x_{14}=06957 ; \\
& x_{21}=01764, x_{22}=05304, x_{23}=15948, x_{24}=00001 ; \\
& x_{31}=00001, x_{32}=00004, x_{33}=00001, x_{34}=13018 ; \\
& z_{11}=82640, z_{12}=13331, z_{13}=8473.70 ; \\
& \widetilde{z_{1}}=(\mathbf{6 9 3 0 9}, \mathbf{8 2 6 4 0}, \mathbf{9 1 1 1 3 . 7 0}) ; \\
& z_{21}=39203, z_{22}=8611, z_{23}=8312.10 ; \\
& \widetilde{z_{2}}=(\mathbf{3 0 5 9 2}, \mathbf{3 9 2 0 3}, \mathbf{4 7 5 1 5 . 1 0}) ;
\end{aligned}
$$

\section{Conclusions}

This work develops an interactive method for solving multi-objective problems with imprecise available supply, forecast demand and unit cost/profit coefficients with triangular possibility distributions. The model designed here aims to simultaneously minimize the total distribu-

Table 2. PIS and NIS values of the objectives.

\begin{tabular}{ccc}
\hline Objective & PIS values & NIS values \\
\hline$z_{11}$ & 81985 & 129630 \\
$z_{12}$ & 18297 & 13263 \\
$z_{13}$ & 8472.50 & 13297 \\
$z_{21}$ & 73447 & 39128 \\
$z_{22}$ & 8475 & 20780 \\
$z_{23}$ & 17187 & 8177.50 \\
\hline
\end{tabular}

tion costs and maximize the total profit with reference to available supply at each source, as well as forecast demand constraints at each destination and the total budget constraint.

The method developed can be easily applied to any real world problem with triangular distributions such as distribution planning decision problems, assignment problems, job allocation problem and more such problems. It provides a systematic framework that facilitates the decision-making process, enabling a DM to interactively modify the imprecise data and related parameters until a set of satisfactory solutions is obtained.

\section{REFERENCES}

[1] L. A. Zadeh, "Fuzzy Sets as a Basis for a Theory of Possibility," Fuzzy Sets and Systems, Vol. 1, No. 1, 1978, pp. 3-28. doi:10.1016/0165-0114(78)90029-5

[2] H.-J. Zimmermann, "Description and Optimization of Fuzzy Systems,” International Journal of General Systems, Vol. 2, No. 1, 1976, pp. 209-215. doi:10.1080/03081077508960870

[3] R. E. Bellman and L. A. Zadeh, "Decision-Making in a Fuzzy Environment,” Management Science, Vol. 17, No. 4, 1970, pp. 141-164.

[4] S. Chanas, W. Kolodziejczyk and A. Machaj, "A Fuzzy Approach to the Transportation Problem," Fuzzy Sets and Systems, Vol. 13, No. 3, 1984, pp. 211-222. doi:10.1016/0165-0114(84)90057-5

[5] H.-J. Zimmermann, "Fuzzy Programming and Linear Programming with Several Objective Functions,” Fuzzy Sets and Systems, Vol. 1, No. 1, 1978, pp. 45-56. doi:10.1016/0165-0114(78)90031-3

[6] L. Li and K. K. Lai, "A Fuzzy Approach to the MultiObjective Transportation Problem,” Computers and $\mathrm{Op}$ erations Research, Vol. 27, No. 1, 2000, pp. 43-57. doi:10.1016/S0305-0548(99)00007-6

[7] M. L. Hussein, “Complete Solutions of Multiple Objective Transportation Problems with Possibilistic Coefficients," Fuzzy Sets and Systems, Vol. 93, No. 3, 1998, pp. 293-299. doi:10.1016/S0165-0114(96)00216-3

[8] W. F. Abd El-Washed, "A Multi-Objective Transportation Problem under Fuzziness," Fuzzy Sets and Systems, Vol. 117, No. 1, 2001, pp. 27-33. doi:10.1016/S0165-0114(98)00155-9

[9] Y. J. Lai, and C. L. Hwang, "A New Approach to Some Possibilistic Linear Programming Problem,” Fuzzy Sets and Systems, Vol. 49, No. 2, 1992, pp. 121-133. doi:10.1016/0165-0114(92)90318-X

[10] T. F. Liang, “Application of Possibilistic Linear Programming to Multi-Objective Distribution Planning Decisions" Journal of the Chinese Institute of Industrial Engineers, Vol. 24, No. 2, 2007, pp. 97-109. doi:10.1080/10170660709509025 